\title{
Physical-Layer Discrimination of Power Line Communications
}

\author{
Brady P. Ross, Timothy J. Carbino, and Samuel J. Stone \\ School of Electrical and Computer Engineering \\ Air Force Institute of Technology \\ WPAFB, OH 45433 \\ Email: [brady.ross, timothy.carbino, samuel.stone]@afit.edu
}

\begin{abstract}
Power Line Communication (PLC) is consistently looked at as a cost effective and elegant solution for many large scale control and automation communication problems. This includes many critical infrastructure Supervisory Control and Data Acquisition (SCADA) systems. Because of this, PLC is investigated for the purposes of Wired Signal Distinct Native Attribute (WS-DNA) PhysicalLayer (PHY) classification and validation as an effective intrusion detection method for PLC networks. This investigation captured intentional PLC messages, extracted WSDNA fingerprints, and utilized Multiple Discriminant Analysis, Maximum Likelihood (MDA/ML) to perform device discrimination. The modulated communication signals of six Insteon Hub devices were investigated to assess the ability to perform device discrimination and validation. For all six devices, an average correct classification of $\% \mathrm{C}=90 \%$ is achieved at Signal to Noise Ratio (SNR) $\geq 40 \mathrm{~dB}$ and \%C $=99 \%$ at $\mathrm{SNR} \geq 46 \mathrm{~dB}$. Receiver Operating Characteristic (ROC) curves are used to illustrate rogue detection results with Equal Error Rate (EER) of $10 \%$ achieved at SNR $=40$ dB.
\end{abstract}

Index Terms-Communications Security, Power Line Communication, Physical-Layer Discrimination, RF-DNA, WS-DNA, Wired Signal.

\section{INTRODUCTION}

In the last several years the number of network devices have increased significantly with the advent of the Internet of Things (IOT). This has placed an increased demand on network security as many of these devices have intermittent connectivity. Media Access Control (MAC) addresses offers a single layer of authentication with it's own strengths and weaknesses. One key weakness is the threat of an attacker spoofing an authentic device in order to steal credentials or other information [1]. A unique solution in the ever growing problem of cyber security and bit level spoofing is to augment bit level credentials with an additional layer of security via Physical-Layer (PHY) discrimination. [2]-[6].

The goal of various PHY fingerprinting investigations in the past has been to improve the verification of devices through the unique physical attributes of their modulated signal [1] [7]. These attempts have focused on the identification of devices based on their radio frequency communication's "unique analog circuitry" [7]. Signal features, or fingerprints, are calculated for regions of interest selected based on signal content within the region [7]. PHY fingerprinting has also been implemented on wired devices in which wired Ethernet is passively tapped [8] and actively tapped [2] for the purpose of creating fingerprints to perform an added layer of network security.
Power Line Communication (PLC) has attracted the attention of industry as an elegant and cost effective solution for large scale control and automation communication networks [9], [10]. This includes Supervisory Control and Data Acquisition (SCADA) networks for smart grids, energy management systems, and other critical infrastructure communications. The appeal of PLC networks is that new infrastructure is usually not required for implementation as the communication lines are already present in the physical powerlines that make up a power grid. PLC networks are yet another example of a technology growing faster than the security designed to protect said technology. Many current PLC networks lack robust intrusion detection and the very nature of the power grid presents PLC as an unmonitored, possibly covert, communications network. Because power supplied by the power grid is required most everywhere, this grants PLC the ability to bypass most current network security measures to extract data from or inject data onto secure systems. To address this problem, the Wired Signal Distinct Native Attribute (WS-DNA) fingerprinting approach along with Multiple Discriminant Analysis, Maximum Likelihood (MDA/ML) are used in an attempt to demonstrate successful PLC device discrimination based on physical signal attributes.

This work is organized as follows. Section II discusses background information and related papers on recent works in device fingerprinting. Section III discusses the methodology and experimental setup, followed by device discrimination results in Section IV and summary and conclusions in Section V.

\section{BACKGROUND}

It is the very entropic nature of the manufacturing processes that introduce distinct variations in electronic components which makes hardware fingerprinting possible. These distinct hardware variations introduce PHY differences in Radio Frequency (RF) emissions that are unique to individual devices and can be used to discriminate devices [11]. These differences can be seen in many hardware components to include capacitors, amplifiers, oscillators, inductors, and other electronic components that effect the fingerprints of each device [11], [12]. The variations in these components in-turn create slight variations in the modulated communication signal which can cause Amplitude Modulation (AM) - Phase Modulation (PM) conversions, alter symbol rates, or alter the observed center frequency [11]. These variations in signals and components also apply to PLC devices. Because of 
this, even if the components are assumed to be identical the imperfections of each device can be exploited to discriminate between devices [3]

Recent fingerprinting work has mostly focused on the intentional emissions of wireless devices [5], [11]-[17]. These research efforts primarily use Radio Frequency Distinct Native Attribute (RF-DNA) feature extraction to create device fingerprints [4]-[6], [17], [18]. The RF-DNA process is adapted herein for use with wired signals and is henceforth refereed to as WS-DNA [19]. MDA/ML is implemented to perform device discrimination based on the WS-DNA fingerprints [5], [17] developed in Section III. This work adopts these techniques and approaches to show that narrow-band PLC devices can be discriminated using WS-DNA generated fingerprints.

\section{A. Power Line Communication (PLC)}

PLC is a method of modulating communications onto the $50 / 60 \mathrm{~Hz}$ sinusoidal AC powerline signal. PLC has long been used by powerline workers to send and received low data rate communications over long distances that can reach over 100 miles without the need of a repeater [20]. Currently, power companies use PLC for one-way communication to perform automatic meter readings as well as in high data rate communications via BroadBand over Powerline (BPL) internet adapters [21]. Home automation communication has also adopted PLC to supplement the control of various sensors and devices. The $\mathrm{X} 10$ protocol was one of the first PLC home automation protocols first used in the early 1980s.

\section{B. Insteon}

Insteon is a manufacturer of home automation devices designed to be networked together via RF, PLC, or a combination of both [22]. The PLC modulated signals sent between Insteon devices will be the target of this research.

Insteon devices communicate via PLC by injecting the communication signal onto the AC powerline [22]. For the United States, household voltage is roughly $120 \mathrm{~V}$ alternating at $60 \mathrm{~Hz}$. Insteon device data, commonly referred to as messages, are modulated using Binary Phase Shift Keying (BPSK) onto a carrier frequency of $131.65 \mathrm{kHz}$ with an average peak-to-peak voltage of $4.64 \mathrm{~V}$ [22]. Instead of 180 degree transitions between bit boundaries, Insteon PLC utilizes a gradual phase shift by introducing 1.5 cycles of carrier at 1.5 times the carrier frequency between bit boundaries. This unique BPSK transition could be a source of additional features that can improve the discrimination of devices. There are two types of PLC messages, standard messages and extended messages, that are broken up into packets with each packet containing 24 bits. The standard messages consist of 5 packets and the extended messages consist of 11 packets [22]. Each packet corresponds to a communication burst that lasts $1.823 \mathrm{~ms}$. A standard message contains a single start packet and four body packets for a total of five packets. An extended message contains a single start packet and 10 body packets for a total of 11 packets. The first 12 bits of a start packet are start/synchronization bits while the remaining 12 are data bits. The first 6 bits of a body packet are start/synchronization bits and the remaining

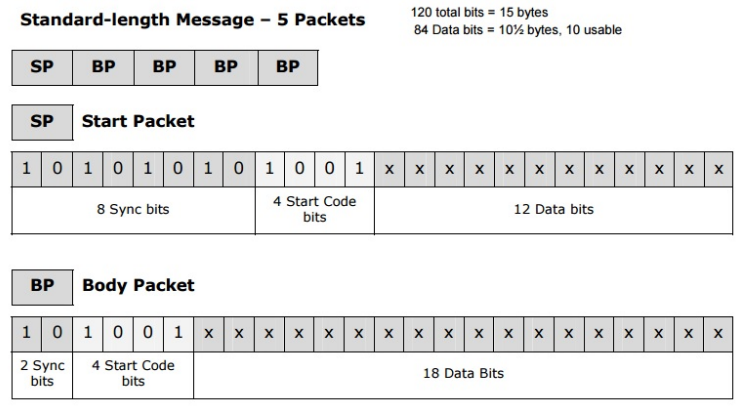

Fig. 1. Insteon Start and Body Packet Structure [22].

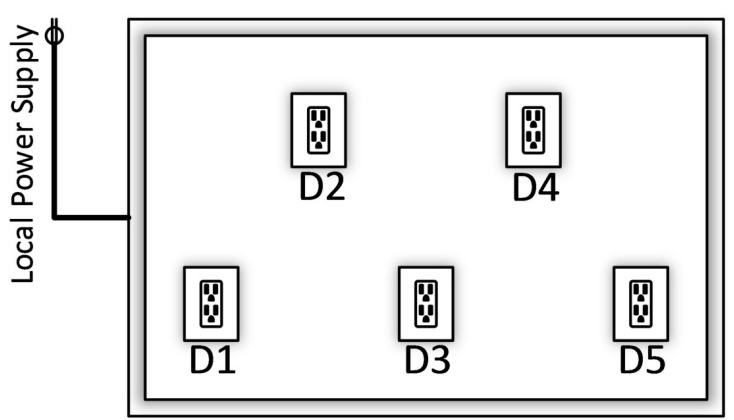

Fig. 2. Experimental Test Fixture Illustration Used for Collections.

18 are data bits. The structure of start and body packets can be seen in Figure 1 [22]. The start of each packet is transmitted $0.876 \mathrm{~ms}$ prior to the zero crossing of the powerline signal and lasts $0.947 \mathrm{~ms}$ after the zero crossing. Each bit is transmitted at a rate of 1 bit every 10 cycles of the carrier frequency, combining this with the 6 zero crossing required for a standard message, the raw bitrate for the Insteon standard message is 2400 bps [22].

\section{EXPERIMENTAL METHOdOLOGY}

This section discusses the collection setup to include the devices under test and their configuration. The methodology specific to WS-DNA is covered and includes the adoption of the RF-DNA processes used herein.

\section{A. Collection Setup and Device Under Test (DUT)}

An $H P{ }^{\circledR}$ Laptop is used to control the collection of the Insteon PLC modulated signals. Insteon for $H U B$ is an application loaded on the laptop and is provided by Insteon to send PLC commands. Two in-line filters are employed to safely condition the signal prior to collection. First, an OnFilter Plug-In Power Line EMI Filter removes the $60 \mathrm{~Hz}$ sinusoidal signal and isolates downstream equipment from the $120 \mathrm{~V}$ household voltage. Second, an anti-aliasing low pass filter with bandwidth of $W_{B}=48 \mathrm{MHz}$ is used to remove any remaining high frequency noise prior to sampling. A LeCroy WavePro 760Zi-A $6.0 \mathrm{GHz}$ oscilloscope operating at a sampling frequency of $f_{s}=10 \mathrm{M}$ Samples/Sec collects and stores the modulated signal for post processing and WS-DNA fingerprint generation.

MATLAB ${ }^{\circledR}$ is used to post process the PLC modulated signals and to generate the WS-DNA fingerprints. During 
TABLE I

DEVICES UNDER TEST

\begin{tabular}{llr}
\multicolumn{3}{c}{ Transmit Devices } \\
\hline Device & Address & Collected SNR \\
\hline Hub 1 & $39: 30: 05$ & $45.2 \mathrm{~dB}$ \\
\hline Hub 2 & $39: 32: 86$ & $45.1 \mathrm{~dB}$ \\
\hline Hub 3 & $39: 21: 82$ & $44.8 \mathrm{~dB}$ \\
\hline Hub 4 & $39: 20: \mathrm{DC}$ & $44.7 \mathrm{~dB}$ \\
\hline Hub 5 & $39: 29: 7 \mathrm{~F}$ & $44.9 \mathrm{~dB}$ \\
\hline Hub 6 & $39: 17: \mathrm{E} 3$ & $45.3 \mathrm{~dB}$ \\
\hline & Receiver Device \\
\hline On/Off Outlet & $39: A 0: 02$
\end{tabular}

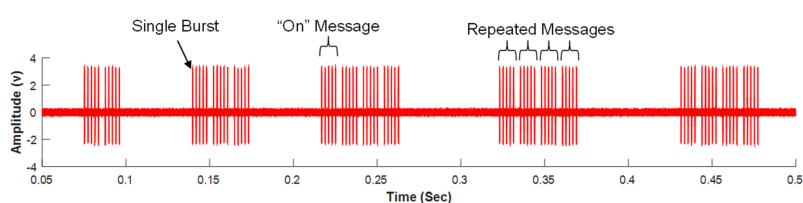

Fig. 3. Typical Time Domain Response of a Modulated PLC On Message Sent From an Insteon Hub Illustrating Bursts, Messages, and Repeated Messages.

this process a 16th order butter-worth software filter centered at $131.65 \mathrm{KHz}$ with a $30 \mathrm{KHz}$ bandwidth is used to process the PLC signals prior to fingerprint generation.

To reduce the potential for outside noise and interference all nearby hardware devices are powered down. However, it is important to determine the effectiveness of WS-DNA and device discrimination in conditions that are less than optimal. Therefore, like-filtered Additive White Gaussian Noise (AWGN) is added to the filtered collected signal to obtain a lower Signal to Noise Ratio (SNR) of $[-10: 50] \mathrm{dB}$ in $2 \mathrm{~dB}$ steps.

The DUT chosen for this analysis is the Insteon Hub that transmits on/off commands to the Insteon On/Off Outlet (receiving device). Multiple PLC On messages sent to the same On/Off Outlet will be collected from six different Insteon Hubs and later used to generate WS-DNA fingerprints. The Hubs and the On/Off Outlet are initially linked according to manufactures direction, however during collection the On/Off Outlet is off network to force the Hub to retransmit messages. This increases the number of messages sent thereby decreasing the time needed to collect a sufficient number for fingerprint generation. Table I summarizes the transmit and receive devices along with estimated collected SNR. To ensure configuration control and integrity of the collection process a test fixture was devised as shown in Figure 2. Each Hub from Table I is individually connected at D1 and the EMI filter at D5 as seen in Figure 2. On messages are then transmitted via the Insteon for $H U B$ application until 400 messages are collected. A single message consists of 5 packets, or bursts, therefore 2000 bursts/packets are collected for each hub device. Figure 3 displays typical $O n$ messages from an Insteon Hub and the repeated messages due to the On/Off Outlet being off network.

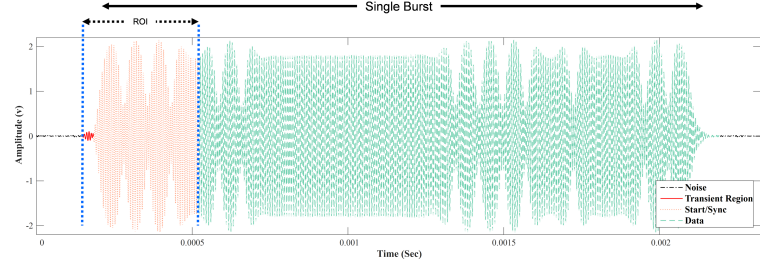

Fig. 4. Typical Time Domain Response of Single Burst From a PLC Modulated On Message Sent From an Insteon Hub. ROI illustrated.

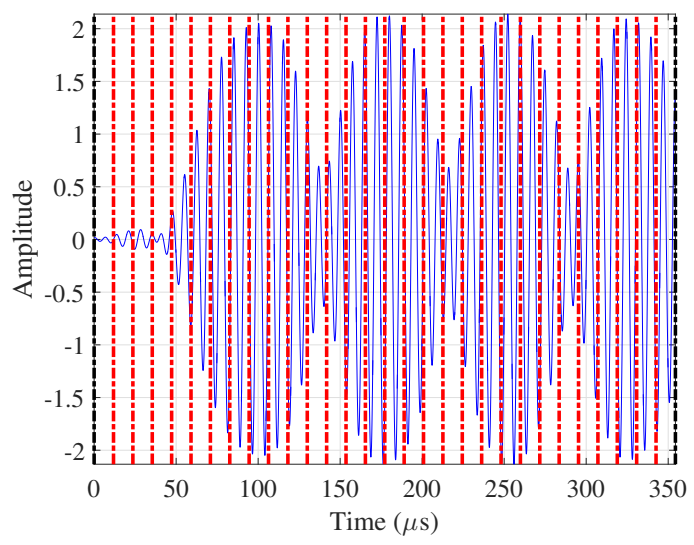

Fig. 5. Representative $353 \mu$ s ROI Corresponding to the First $303 \mu \mathrm{s}$ of a Burst From a PLC Modulated Insteon Hub On Message, Including $50 \mu$ s Transient Ramp Up Region, Divided into $N_{\text {reg }}=30$ Subregions.

\section{B. WS-DNA Fingerprinting}

The approaches and processes used in WS-DNA Fingerprinting are adapted from RF-DNA literature [4]-[6], [17], [18]. As presented in Section II-B each of the 5 bursts/packets in a message contain symbols that correspond to start/sync bits. These start/sync bits within messages are a steady state response and do not change from one device to another. Referring to Figure 1, the first 4 symbols of any burst/packet will always be the same no matter what message is sent, or what the sender/receiver is. Figure 4 displays an illustration of this region including what appears to be a transient ramp up period that occurs approximately $50 \mu s$ before the first symbol is transmitted. The region that contains these start/sync symbols along with the transient ramp up period is the Region Of Interest (ROI) and corresponds to first $353 \mu s$ of each burst. The ROI is separated into $N_{\text {reg }}=30$ equally spaced, adjoining sub-regions. Each sub-region is compromised of $n$ discrete time samples and can be seen in Figure 5. A total of, $N_{\text {Resp }}=3$ Instantaneous responses to include 1) amplitude $\{a(n)\}, 2)$ phase $\{\phi(n)\}$, and 3) frequency $\{f(n)\}$ are calculated for each of the $N_{\text {Reg }}=30$ subregions of the ROI. For each of these instantaneous responses, $N_{\text {Stat }}=4$, statistical features are created including: 1) standard deviation $(\sigma), 2)$ variance $\left.\left(\sigma^{2}\right), 3\right)$ skewness $(\gamma)$, and 4) kurtosis $(\kappa)$. Together with the entire ROI, there are $N_{\text {treg }}=N_{\text {reg }}+1=31$ total sub-regions that are used to form the regional fingerprint vectors:

$$
F_{R_{i}}=\left[\begin{array}{llll}
\sigma_{R_{i}} & \sigma_{R_{i}}^{2} & \gamma_{R_{i}} & \kappa_{R_{i}}
\end{array}\right]_{1 \times 4},
$$


where $i=1,2, \ldots, N_{\text {treg }}$ [4]. Each vector from (1), are concatenated to form each of the instantaneous response vectors:

$$
\boldsymbol{F}_{I_{a, \phi, f}}=\left[\begin{array}{lllllll}
F_{R_{1}} & \vdots & F_{R_{2}} & \vdots & F_{R_{3}} & \ldots & F_{R_{N_{\text {treg }}}}
\end{array}\right]_{1 \times 4\left(N_{\text {treg }}\right)},
$$

where the $I$ denotes each instantaneous response $\{a(n)\}$, $\{\phi(n)\}$, and $\{f(n)\}$ [4]. For each instantaneous response vector, the final composite WS-DNA fingerprint vector is created as:

$$
\boldsymbol{F}_{\boldsymbol{C}}=\left[\begin{array}{lllll}
\boldsymbol{F}_{a} & \vdots & \boldsymbol{F}_{\boldsymbol{\phi}} & \vdots & \boldsymbol{F}_{f}
\end{array}\right]_{1 \times 4\left(N_{\text {treg }}\right) \times 3},
$$

resulting in fingerprints with at total of $N_{\text {feats }}=3 \times 4 \times$ $31=372$ features [4].

\section{Device Discrimination}

Five different Monte Carlo noise realizations are added to each of the experimentally collected realizations of each SNR step prior to fingerprint generation. As mentioned in Section III-A, SNR is varied from $-10 \mathrm{~dB}$ to $50 \mathrm{~dB}$ in $2 \mathrm{~dB}$ steps to simulate different channel noise conditions and to evaluate the process using degraded signals. Note, the way the SNR is varied does not suggest we improved SNR levels above the estimated collected SNR of the signal shown in Table I. The $S N R_{\text {Sim }}$ is calculated by considering all collected signal and noise as contributing to signal power with the only noise contribution comprised of the AWGN component. This is a conservative estimate of SNR as the actual SNR is likely lower than $S N R_{\text {Sim }}$ as the collected signal does include some noise.

MDA/ML classification was conducted for 6 classes/devices with the number of Training fingerprints equal to the number of Testing fingerprints per each class [3], [4]. A total of 2000 fingerprints were collected per class with five different noise realizations at each SNR level for a total of $F=10000$ fingerprints in the WS-DNA process mentioned previously. Thus, $F_{t r n}=5000$ fingerprints were used for Training and $F_{t s t}=5000$ fingerprints used for Testing. K-fold cross validation is used during Training to increase reliability. An appropriate value of $\mathrm{K}=5$ is chosen consistent with common statistical practice [23]. Device discrimination will be assessed with an average percent correct curve, a confusion matrix, and Receiver Operating Characteristic (ROC) curves presented in section IV.

\section{RESULTS}

Discrimination results for 6 devices shown in Table I are presented. These discrimination results are consistent with the RF-DNA fingerprint and MDA/ML processes discussed in section III [4]-[6], [17], [18]. A total of 1000 fingerprints were used for Testing with five different noise realizations equating to 5000 fingerprints per device. Figure 6 displays WS-DNA fingerprinting results for the 6 devices in average percent correct $(\% \mathrm{C})$ vs SNR. For all devices, $\% \mathrm{C}=90 \%$ is achieved at $\mathrm{SNR} \geq 40 \mathrm{~dB}$ and $\% \mathrm{C}=99 \%$ at $\mathrm{SNR} \geq 46 \mathrm{~dB}$. Table II gives a confusion matrix displaying $\% \mathrm{C}$ for $\mathrm{SNR}=40 \mathrm{~dB}$ and $30 \mathrm{~dB}$. This shows that Hubs 1, 5, 6 are more often misclassified with one another than the other Hubs. This could be due to

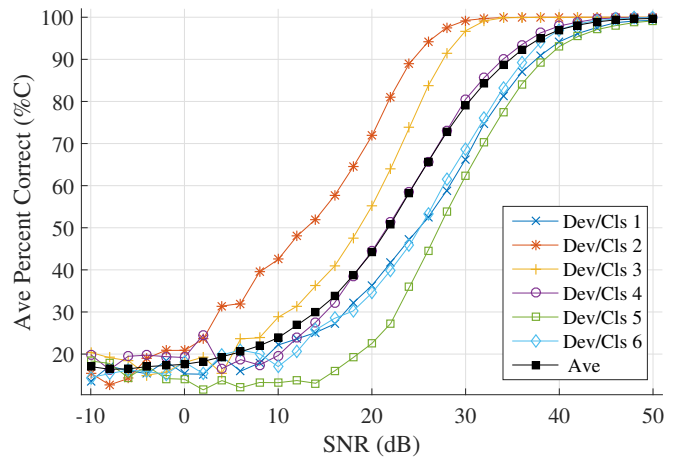

Fig. 6. Device Classification Results for 6 Different Insteon Hubs with Average Percent Correct (\%C) with Varying Simulated SNR levels.

TABLE II

CONFUSION MATRIX FOR WS-DNA FINGERPRINTING AT SNR $=40$ $d \mathrm{~B}$ AND SNR $=30 d \mathrm{~B}$ WITH 5000 TRIALS PER DEVICE. RESUlTS ARE PRESENTED AS \% C AT $40 d \mathrm{~B} / 30 d \mathrm{~B}$.

\begin{tabular}{|c|c|c|c|c|c|c|}
\hline & Hub 1 & Hub 2 & Hub 3 & Hub 4 & Hub 5 & Hub 6 \\
\hline Hub 1 & $94.19 / 66.31$ & $0 / 0$ & $0 / .20$ & $0 / .16$ & $3.47 / 17.19$ & $2.34 / 16.14$ \\
\hline Hub 2 & $0 / 0$ & $100.00 / 99.24$ & $0 / .40$ & $0 / .36$ & $0 / 0$ & $0 / 0$ \\
\hline Hub 3 & $0 / .02$ & $.02 / .90$ & $99.98 / 96.78$ & $0 / .85$ & $0 / 87$ & $0 / .58$ \\
\hline Hub 4 & $0 / .32$ & $0 / 98$ & $0 / .95$ & $97.93 / 80.52$ & $2.07 / 17.01$ & $0 / .22$ \\
\hline Hub 5 & $3.34 / 11.14$ & $0 / 0$ & $0 / 86$ & $2.25 / 15.48$ & $93.04 / 62.33$ & $1.37 / 10.19$ \\
\hline Hub 6 & $1.85 / 14.95$ & $0 / 0$ & $0 / .56$ & $0 / .14$ & $1.12 / 15.60$ & $97.03 / 68.75$ \\
\hline
\end{tabular}

higher similarities in subcomponents of the devices when compared with the others.

To assess rogue detection, ROC curves are used to display how often rogue devices are rejected and authentic devices are accepted at specific SNRs. A benchmark of Equal Error Rate $(E E R)=10 \%$ is established, i.e, $90 \%$ of unauthorized rogue devices are successfully rejected and $90 \%$ of authorized devices are accepted. To simulate a rogue device attempting access, fingerprints already created for Hub 4 are used for simulated intrusion detection. Figure 7 displays the rogue detection ROC curve with $\mathrm{SNR}=30 \mathrm{~dB}$. An EER $=10 \%$ is achieved for all devices except Hub 5 at $30 \mathrm{~dB}$. The ROC curves in Figure 8 show rogue detection results of EER $=10 \%$ for all devices with $\mathrm{SNR}=40 \mathrm{~dB}$.

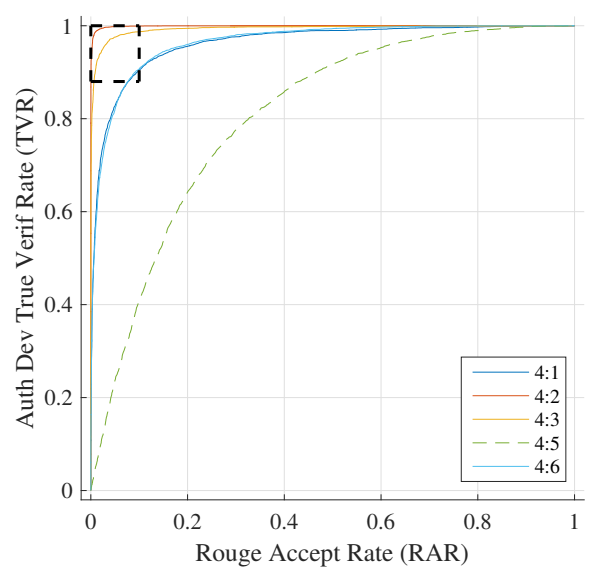

Fig. 7. ROC Curve for Rouge Detection with Hub 4 Representing Rouge Device at SNR $=30 \mathrm{~dB}$, with EER $=10 \%$ illustrated by dashed box. 


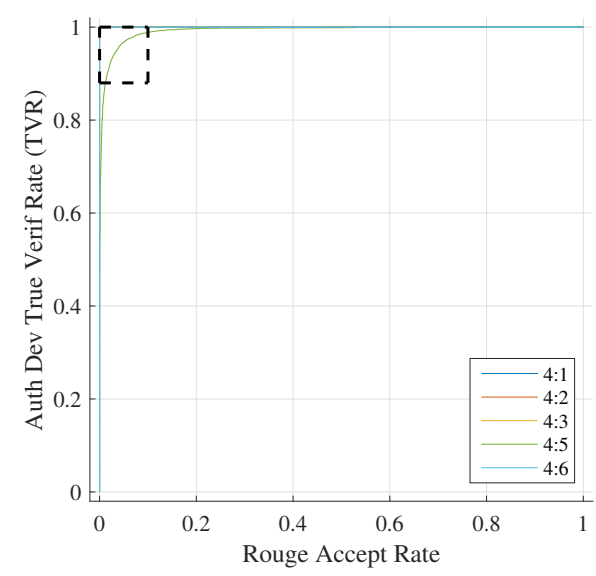

Fig. 8. ROC Curve for Rouge Detection with Hub 4 Representing Rouge Device at $\mathrm{SNR}=40 \mathrm{~dB}$, with EER $=10 \%$ illustrated by dashed box.

\section{CONCLUSIONS}

Performance for PHY device discrimination of PLC modulated signals based on WS-DNA fingerprints is presented in this paper. The WS-DNA fingerprinting process was applied to 6 different Insteon Hub devices with MDA/ML generated discrimination results.

Average correct classification performance was acceptable with $\% \mathrm{C}=90 \%$ at $\mathrm{SNR} \geq 40 \mathrm{db}$. This is well below the collected SNR of approximately $45 \mathrm{~dB}$. The benchmark $10 \%$ EER for rogue intrusion detection was achieved at $\mathrm{SNR}=40 \mathrm{~dB}$, again below the collected SNR. These results show that an additional layer of security for PLC modulated signals based on WS-DNA fingerprinting is possible. The results of this research provide evidence that a 2-factor security authentication (WS-DNA and bitlevel credentials) will vastly improve the systems intrusion detection/prevention.

There remains significant research on the topic of WSDNA as applied to PLC devices. As MDA/ML was the process used for discrimination of the generated fingerprints, alternative discriminators can be investigated. Using Generalized Relevance Learning Vector QuantizedImproved (GRLVQI) as a discriminator would an obvious next step. GRLVQI provides a better illustration of feature relevance ranking on decisions made by the classifiers [17]. An increase in feature relevance can improve performance and the possibility of real world application raises greatly [8]. Future work can also include specifically targeting the symbol boundaries. These transition areas could be rich with device specific features that can improve performance.

\section{REFERENCES}

[1] Y. Sheng, K. Tan, G. Chen, D. Kotz, and A. Campbell, "Detecting 802.11 mac layer spoofing using received signal strength," in INFOCOM 2008. The 27th Conference on Computer Communications. IEEE, April 2008.

[2] M. M. R. S. F. . D. T. E. Gerdes, R. M., "Physical-layer identification of wired ethernet devices." Information Forensics and Security, IEEE Transactions on, vol. 7, no. 4, p. 6, 2012
[3] W. E. Cobb, E. D. Laspe, R. O. Baldwin, M. A. Temple, and Y. C. Kim, "Intrinsic physical-layer authentication of integrated circuits," IEEE Transactions on Information Forensics and Security, vol. 7, no. 1, pp. 14-24, Feb 2012.

[4] W. E. Cobb, E. W. Garcia, M. A. Temple, R. O. Baldwin, and Y. C. Kim, "Physical layer identification of embedded devices using rf-dna fingerprinting," in MILITARY COMMUNICATIONS CONFERENCE, 2010 - MILCOM 2010, Oct 2010, pp. 2168-2173.

[5] B. W. Ramsey, M. A. Temple, and B. E. Mullins, "Phy foundation for multi-factor zigbee node authentication," in Global Communications Conference (GLOBECOM), 2012 IEEE, Dec 2012, pp. 795-800.

[6] M. D. Williams, S. A. Munns, M. A. Temple, and M. J. Mendenhall, "Rf-dna fingerprinting for airport wimax communications security," in Network and System Security (NSS), 2010 4th International Conference on, Sept 2010, pp. 32-39.

[7] D. Z. Danev, Boris and S. Capkun, "On physical-layer identification of wireless devices." ACM Computing Surveys (CSUR), vol. 45, no. 1 , p. 6, 2012

[8] T. J. Carbino, M. A. Temple, and J. Lopez Jr, "A comparison of phy-based fingerprinting methods used to enhance network access control," in IFIP International Information Security Conference. Springer, 2015, pp. 204-217.

[9] G. Bumiller, L. Lampe, and H. Hrasnica, "Power line communication networks for large-scale control and automation systems," IEEE Communications Magazine, vol. 48, no. 4, pp. 106-113, April 2010.

[10] C. Semiconductor, "What is power line communication," EE Times, vol. 8, p. 17, 2011

[11] Y. Huang and H. Zheng, "Radio frequency fingerprinting based on the constellation errors," in 2012 18th Asia-Pacific Conference on Communications (APCC), Oct 2012, pp. 900-905.

[12] V. Brik, S. Banerjee, M. Gruteser, and S. Oh, "Wireless device identification with radiometric signatures," in Proceedings of the 14th ACM International Conference on Mobile Computing and Networking, ser. MobiCom '08. New York, NY, USA: ACM, 2008, pp. 116-127. [Online]. Available: http://doi.acm.org/10.1145/1409944.1409959

[13] B. Danev, D. Zanetti, and S. Capkun, "On physical-layer identification of wireless devices," ACM Comput. Surv., vol. 45, no. 1, pp. 6:1-6:29, Dec. 2012. [Online]. Available: http://doi.acm.org/10.1145/2379776.2379782

[14] A. Candore, O. Kocabas, and F. Koushanfar, "Robust stable radiometric fingerprinting for wireless devices," in Hardware-Oriented Security and Trust, 2009. HOST '09. IEEE International Workshop on, July 2009, pp. 43-49.

[15] M. Edman and B. Yener, "Active attacks against modulationbased radiometric identification. rensselaer institute of technology," Technical report, Tech. Rep., 2009.

[16] B. Danev, H. Luecken, S. Capkun, and K. El Defrawy, "Attacks on physical-layer identification," in Proceedings of the Third ACM Conference on Wireless Network Security, ser. WiSec '10. New York, NY, USA: ACM, 2010, pp. 89-98. [Online]. Available: http://doi.acm.org/10.1145/1741866.1741882

[17] D. R. Reising, M. A. Temple, and M. E. Oxley, "Gabor-based rf-dna fingerprinting for classifying 802.16e wimax mobile subscribers," in Computing, Networking and Communications (ICNC), 2012 International Conference on, Jan 2012, pp. 7-13.

[18] M. D. Williams, M. A. Temple, and D. R. Reising, "Augmenting bit-level network security using physical layer rf-dna fingerprinting," in Global Telecommunications Conference (GLOBECOM 2010), 2010 IEEE, Dec 2010, pp. 1-6.

[19] J. Lopez Jr, M. A. Temple, and B. E. Mullins, "Exploitation of hart wired signal distinct native attribute (ws-dna) features to verify field device identity and infer operating state," in International Conference on Critical Information Infrastructures Security. Springer, 2014, pp. 24-30.

[20] S. H. Horowitz and A. G. Phadke, Power system relaying. John Wiley \& Sons, 2008, vol. 22.

[21] J. Newbury, "Communication requirements and standards for low voltage mains signalling," IEEE transactions on power delivery, vol. 13, no. 1, pp. 46-52, 1998.

[22] "Insteon whitepaper: The details," Insteon, Tech. Rep., 2013.

[23] J. Friedman, T. Hastie, and R. Tibshirani, The elements of statistical learning. Springer series in statistics Springer, Berlin, 2001, vol. 1. 